\title{
A novel compound mutation in GLRA1 cause hyperekplexia in a Chinese boy- a case report and review of the literature
}

\author{
Zhiliang Yang ${ }^{*}$ (D), Guilian Sun, Fang Yao, Dongying Tao and Binlu Zhu
}

\begin{abstract}
Background: The pathogenesis of hereditary hyperekplexia is thought to involve abnormalities in the glycinergic neurotransmission system, the most of mutations reported in GLRA1. This gene encodes the glycine receptor a1 subunit, which has an extracellular domain (ECD) and a transmembrane domain (TMD) with 4 a-helices (TM1-TM4).

Case presentation: We investigated the genetic cause of hyperekplexia in a Chinese family with one affected member. Whole-exome sequencing of the 5 candidate genes was performed on the proband patient, and direct sequencing was performed to validate and confirm the detected mutation in other family members. We also review and analyse all reported GLRA1 mutations. The proband had a compound heterozygous GLRA1 mutation that comprised 2 novel GLRA1 missense mutations, C.569C > T (p.T190 M) from the mother and C.1270G > A (p.D424N) from the father. SIFT, Polyphen-2 and MutationTaster analysis identified the mutations as disease-causing, but the parents had no signs of hyperekplexia. The p.T190 M mutation is located in the ECD, while p.D424N is located in TM4.
\end{abstract}

Conclusions: Our findings contribute to a growing list GLRA1 mutations associated with hyperekplexia and provide new insights into correlations between phenotype and GLRA1 mutations. Some recessive mutations can induce hyperekplexia in combination with other recessive GLRA1 mutations. Mutations in the ECD, TM1, TM1-TM2 loop, TM3, TM3-TM4 loop and TM4 are more often recessive and part of a compound mutation, while those in TM2 and the TM2-TM3 loop are more likely to be dominant hereditary mutations.

Keywords: Hyperekplexia, Startle disease, GLRA1, Phenotype

\section{Background}

Hyperekplexia, also known as startle disease, is a rare disorder that is classically characterized by exaggerated startle responses to unexpected stimuli. It was first reported in 1958 [1]. This disorder can cause serious injuries due to frequent falls and may cause infantile death via induced apnoea. Hyperekplexia can be hereditary or can occur sporadically. It shows genetic heterogeneity, with the first causal mutations reported in GLRA1 (glycine receptor (GlyR) alpha 1) in 1993 [2]. Mutations in 4 other genes have since been reported: GLRB (GlyR beta) [3-8], GPHN (gephyrin) [9], ARHGEF9 (Cdc42 guanine nucleotide exchange factor 9) [10]

\footnotetext{
* Correspondence: sizhewujiu@163.com

Department of Pediatrics, The First Hospital of China Medical University, No.

155 Nanjing North Street, Heping District, Shenyang 110001, Liaoning

Province, People's Republic of China
}

and SLC6A5 (solute carrier family 6 member 5) [11-14]. All of these genes encode proteins that are associated with the glycine transmission system. Recently, a clear correlation of mutation in $\beta$-catenin gene (CTNNB1) with an atypical syndromic hyperekplexia had been reported in a case of CTNNB1-related syndrome and CTNNB1 was considered to be a cause gene for syndromic hyperekplexia [15].

The most of mutations have been reported in GLRA1 $[16,17]$, which encodes the GlyR $\alpha 1$ subunit. This subunit contains an extracellular domain (ECD) and a transmembrane domain (TMD) that comprises $4 \alpha$-helices, termed TM1-TM4. The mutation loci have been reported to be associated with recessive or dominant heredity [17]. Here we report a case of genetically confirmed hyperekplexia caused by two novel GLRA1 mutations, which together constitute a compound 
mutation, that were inherited from the proband's unaffected parents. We also discuss possible correlations between mutation loci and hyperekplexia phenotype.

\section{Case presentation}

The proband (Fig. 1a) was a 13-year-old Chinese boy who was admitted to hospital with a chief complaint of frequent falls in response to sudden stimuli for about 13 years. He was born at term and had an unremarkable antenatal and birth history. In the neonatal period, he showed body rigidity and trembling for a few seconds in response to vocal stimulation but did not show apnoea, and his parents paid little attention at the time. He showed no developmental delays or neurologic deficits, but seemed "timid" when he began to walk at age 1 year and showed body stiffening in response to sudden audible, visual or tactile stimulation, sometimes falling down and becoming pale but without losing consciousness. $\mathrm{He}$ often had superficial facial injuries in kindergarten and in primary school. When he was 10 years old, he fell and broke his right forearm, and twice he fell down stairs in response to sudden noises. After that, he was accompanied by a family member when he went outside or walked up and down stairs. About 1 month prior to admission, he fell after being startled and broke his left forearm.

The boy was admitted to several other hospitals over the years. His brain MRI and MRA, 24-h electroencephalogram (EEG), electrocardiogram (ECG) and transcranial Doppler ultrasound apparatus (TCD) results were normal. He was diagnosed with possible epilepsy and prescribed antiepileptics; however, he showed no improvement after 3 months, so the antiepileptics were withdrawn. Physical examination showed a cautious gait with a wide stride, and the nose-tapping test (head-retraction reflex) was positive. The IQ (intelligence quotient) using the Wechsler Intelligence Scale for Children was 89. To exclude epilepsy, a video EEG was performed. During the test, some stimuli were presented, but no abnormal waves were induced. His ceruloplasmin and lactic acid levels were normal. Hyperekplexia was considered based on his medical history and on repeated normal testing results from several hospitals.

After genetic counselling with a clinical geneticist, genetic analysis for hyperekplexia was performed after obtaining the approval of the ethics committee of the First Hospital of China Medical University. With written consent from his parents, peripheral blood samples were collected from the proband and his parents. DNA was extracted using the Puregene Extraction Kit (Qiagen, Germany).The Agilent SureSelect Human Exon Capture Sequencing platform was used to screen DNA from the proband for the 5 genes involved in the glycinergic neurotransmission system on DNA i.e. GLRA1, GLRB, GPHN, ARHGEF9 and SLC6A5.The obtained data were analysed using Agilent SureSelect Human All Exon V5 software, and the variants were called according to the protocol for the platform. The variants were interpreted according to the guidelines from American College of Medical Genetics and Genomics and patient phenotype [18]. The detected missense mutations were validated using direct sequencing. Direct sequencing was performed on DNA from individuals of all family members using the 3730xl DNA Analyzer (Applied Biosystems, Foster City, CA, USA), and the samples were subjected to sequence analysis using Sequence Scanner v1.0 (Applied Biosystems, Foster City, CA, USA). The sequencing procedure or mutation validation were performed by Hicetech Test Laboratory (Beijing, China) which provides the third party inspection services.

The hypothetical effects of the mutations on protein function were analysed using the Polymorphism Phenotyping v2

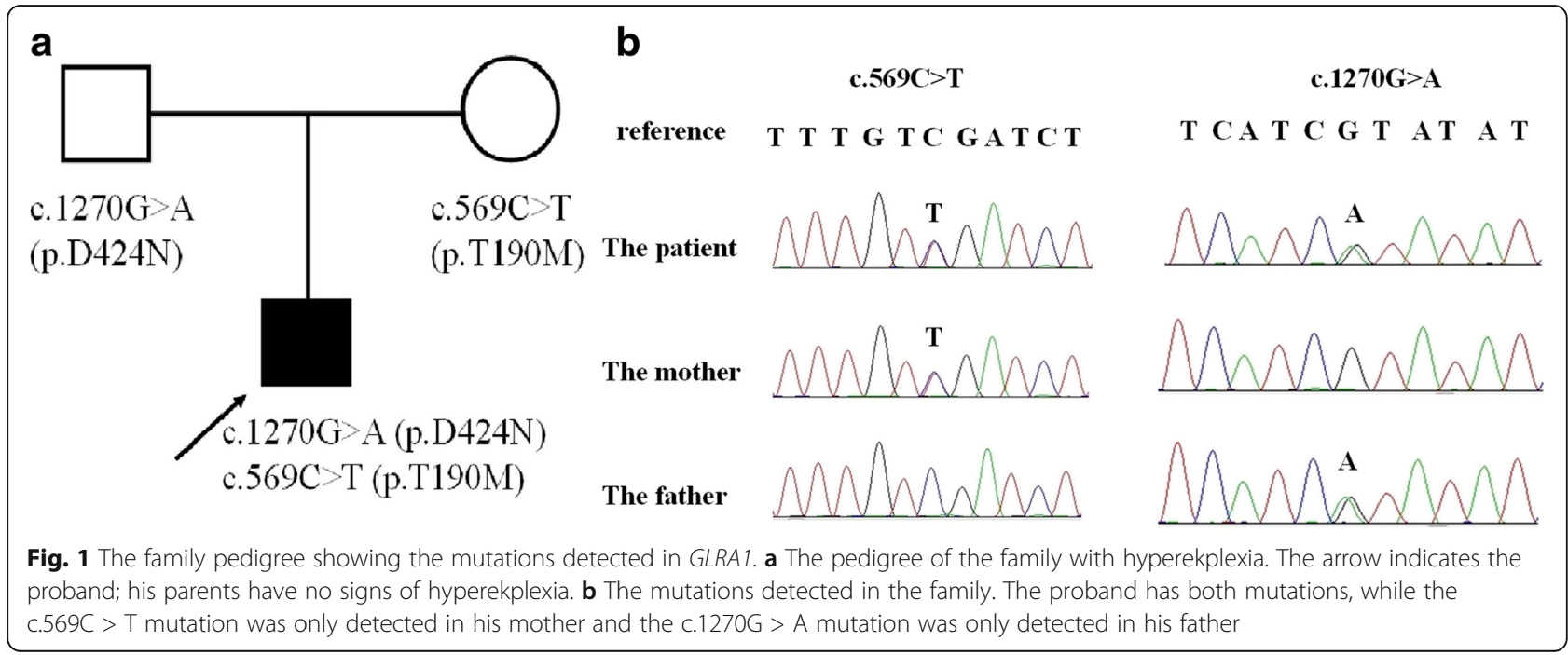


(PolyPhen-2) prediction tool (http://genetics.bwh.harvard.edu/pph2/dbsearch.shtml), SIFT (http://sift.jcvi.org/ www/SIFT_enst_submit.html) and MutationTaster (http://www.mutationtaster.org/index.html).

Almost all hyperekplexia cases respond well to clonazepam (CZP). CZP is a $\gamma$-aminobutyric acid receptor alpha1(GABARA1) agonist and can enhance GABA-gated chloride channel function. The glycine receptor and GABA receptor are members of same superfamily of ligand-gated ion channels and share common transmembrane topology, structural and functional features. CZP was presumed to compensate for the defective glycine-gated chloride channel function by enhancing GABA-gated chloride channel function in hyperekplexia [19].

In our patient, after the GLRA1 mutations were identified and hyperekplexia was diagnosed, CZP administration was initiated at $0.02 \mathrm{mg} / \mathrm{kg}$ per day, administered in 3 doses, with slight increases every 3 day. After 1 week, the daily dose was $0.05 \mathrm{mg} / \mathrm{kg}$, and his parents reported that the startle response had almost disappeared, but the patient appeared sleepy all day. The dose of CZP was unchanged, and at the 6-month follow-up, the startle responses were almost resolved.

The proband patient had 2 heterozygous missense point mutations in GLRA1 (C.569C > T (p.T190 M) and C.1270G > A (p.D424N) (reference sequence: NM_001146040) that were validated with direct sequencing. His mother had the C.569C > T (p.T190 M) mutation, and his father had the C.1270G > A (p.D424N) mutation (Fig. 1b). The p.T190 M mutation is located in the ECD, while p.D424N is located in TM4. PolyPhen-2, SIFT and MutationTaster analysis suggested that both of these mutations would negatively affect gene function (Table 1 ).

We next reviewed reported GLRA1 mutations in hyperekplexia according to mutation location (Table 2). About $67.7 \%(42 / 62)$ are recessive, about 29\% (18/62) are dominant and about $3.2 \%(2 / 62)$ are novel mutations. Among the recessive mutations, about 52.4\% (22/ 42) are compound mutations (i.e. present in combination with another mutation). The compound mutation in our patient was first reported, we cannot define whether it is dominant or recessive. The mutations were mostly located in the ECD (21/62) and TM2 (13/62), with only 1 mutation in the TM1-TM2 loop and 2 in TM3. About $90.4 \%(19 / 21)$ of the mutations in the ECD domain, about $30.8 \%$ in the TM2 domain, all in TM3 and the TM3-TM4 loop, and 75\% in TM4 were recessive. The only mutation in the TM1-TM2 loop was dominant; while $69.2 \%$ of the mutations in the TM2 domain and about $66.7 \%$ in the TM2-TM3 loop were dominant (Fig. 2). The mutations and referenced studies are shown in Additional file 1: Table S1.

\section{Discussion and conclusions}

Mutations in 5 candidate genes relating to the glycinergic neurotransmission system have been identified in hyperekplexia [2-14]). The GLRA1 gene encodes the GlyR $\alpha 1$ subunit [2], GPHN encodes gephyrin [9], $A R H$ GEF9 encodes collybistin [10] and SLC6A5 encodes the glycine transporter GlyT2 [11]. Gephyrin and collybistin are involved in GlyR clustering, while the glycine transporter is a presynaptic $\mathrm{NaCl}$-dependent transporter. Some gene-negative cases in the 5 genes have been reported, raising the possibility that there are other candidate genes [20], and CTNNB1 was reported recently [15], the event indicate hyperekplexia could involve multiple genes.

We reviewed all of the GLRA1 mutations in hyperekplexia that have been reported to date and found that $29 \%$ are dominant missense and $67.7 \%$ are recessive missense mutations. This is in accordance with a report that $23 \%$ of the mutations are dominant missense, $39 \%$ are recessive missense and $38 \%$ are recessive nonsense [20]. Our review found that mutations more often occurred in the ECD and TM2 domains; that mutations in the ECD, TM1, TM1-TM2 loop, TM3, TM3-TM4 loop and TM4 were more likely to be recessive and compound mutations (with other heterozygous mutations); and that few mutations were located in TM2 and the TM2-TM3 loop, but these were more likely to be dominant pathogenic mutations.

Our patient had two heterozygous mutations, C.569C > T(p.T190 M) from his mother, located in the ECD, and C.1270G > A(p.D424N) from his father, located in TM4. Neither were located in regions of the gene in which they were likely to be dominant hereditary mutations. Indeed, neither parent had hyperekplexia, even in silico analysis predicted that each mutation would be disease causing. However, the proband patient, who had both mutations, had hyperekplexia. This suggests that these recessive mutations of the GLRA1 gene in a compound heterozygote state are pathogenic and cause hyperekplexia.

Table 1 Functional evaluation of the GLRA1 mutations detected in the family of a Chinese boy with hyperekplexia

\begin{tabular}{llllll}
\hline Base change & Exon number & Amino acid change & PolyPhen-2 analysis & SIFT analysis & MutationTaster analysis \\
\hline c.569C $>$ T & 6 & p.T190M & Probably damaging & Damaging & Disease causing \\
c.1270G $>$ A & 9 & p.D424N & Probably damaging & Damaging & Disease causing \\
\hline
\end{tabular}


Table 2 Distribution of hyperekplexia mutations in GLRA1 according to the hGlyR position

\begin{tabular}{|c|c|c|c|c|c|}
\hline hGlyR position & $\begin{array}{l}\text { Mutations } \\
n\end{array}$ & $\begin{array}{l}\text { Recessive mutations } \\
n(\%)\end{array}$ & $\begin{array}{l}\text { Recessive mutations that } \\
\text { are compound mutations } \\
n(\%)\end{array}$ & $\begin{array}{l}\text { Reported dominant mutations } \\
n(\%)\end{array}$ & $\begin{array}{l}\text { Reported de novo mutations } \\
n(\%)\end{array}$ \\
\hline NA & 2 & $2(100)$ & $1(50)$ & 0 & 0 \\
\hline ECD & 21 & 19 (90.4) & $10(52.6)$ & $1(4.8)$ & $1(4.8)$ \\
\hline TM1 & 7 & $5(71.4)$ & $2(40)$ & $2(28.6)$ & 0 \\
\hline TM1-TM2 loop & 1 & 0 & - & $1(100)$ & 0 \\
\hline TM2 & 13 & $4(30.8)$ & $1(25)$ & $9(69.2)$ & 0 \\
\hline TM2-TM3 loop & 6 & $1(16.7)$ & 0 & $4(66.7)$ & $1(16.7)$ \\
\hline TM3 & 2 & $2(100)$ & $2(100)$ & 0 & 0 \\
\hline TM3-TM4 loop & 6 & $6(100)$ & $4(66.7)$ & 0 & 0 \\
\hline TM4 & 4 & $3(75)$ & $2(66.7)$ & $1(25)$ & 0 \\
\hline Total & 62 & $42(67.7)$ & $22(52.4)$ & $18(29)$ & $2(3.2)$ \\
\hline
\end{tabular}

$N A$ not applicable, ECD extracellular binding domain, $T M$ transmembrane domain, $n$ number of mutations, \% percentage of mutations

The missense heterozygous mutations in the parents of the proband were autosomal recessive, even though the analysis found that they could cause abnormal protein function. It is possible that having a normal allele produces sufficient protein to allow normal glycinergic neurotransmission. When the patient inherited both mutations from his parents, the deleterious effects of the mutated proteins could not be counteracted by an unaffected allele.

Some mutant proteins are recognized by the endoplasmic reticulum (ER) control system and broken down via proteasomes [21]. The mutated proteins were not released into the blood and were not active, which could explain why the mutations are recessive hereditary mutations. If the mutated proteins bypass the ER control system, they are transported into the blood and trigger

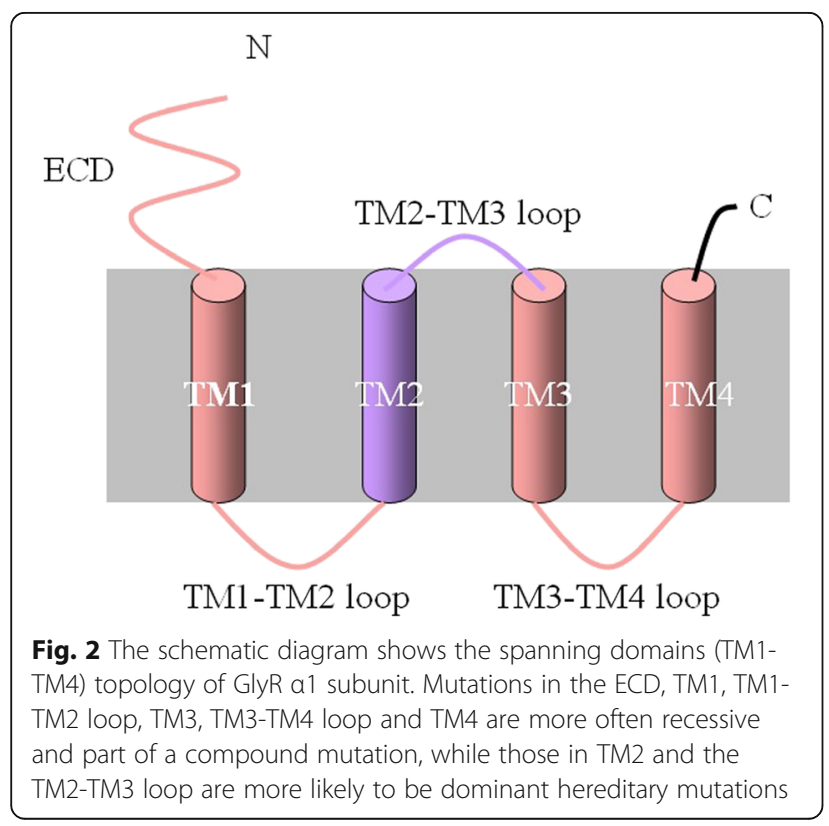

an autoimmune response. Notably, GAD65 (glutamic acid decarboxylase 65-kilodalton isoform, glutamate decarboxylase 2) and GlyR autoimmunity may result in stiff-person syndrome or in progressive encephalomyelitis with rigidity and myoclonus (PERM) [22]. A striking feature of PERM with GlyR autoantibodies is a pathologically exaggerated startle response that resembles hyperekplexia [23]. We did not test our patient for relevant autoantibodies as we did not have access to the technology.

In conclusion, hyperekplexia is potentially treatable, and should be treated in order to prevent injuries and improve the quality of life. Early diagnosis and treatment is important. The location of the GLRA1 mutation correlates hyperekplexia, and gene-negative cases of hyperekplexia suggest that other genes may be involved in this disorder. Although hyperekplexia is a clinical diagnosis, we suggest that prompt genetic analysis may be useful for early definite diagnosis of hyperekplexia and subsequent preconception counselling and safer care for affected neonates.

\section{Additional file}

Additional file 1: Table S1. List of hyperekplexia mutations in GLRA1 according to the hGlyR position. References of individual studies for mutations in GLRA1 gene are summarized in the Supplementary Table. NA, not applicable; ECD extracellular binding domain; TM,

transmembrane domain; n, number of mutations. (DOC 306 kb)

\section{Abbreviations}

CZP: Clonazepam; ECD: Extracellular domain; GABA: $y$-Aminobutyric acid; GlyR: Glycine receptor; TMD: Transmembrane domain

Acknowledgements

The authors thank the family for participating and supporting this study.

Funding

No funding was obtained for this study. 


\section{Availability of data and materials}

The data of the current study are available from the corresponding author on reasonable request

\section{Authors' contributions}

ZLY was responsible for acquisition of the clinical information and writing up and reviewing the manuscript. FY, DYT and BLZ assisted in developing figures, images and table for the manuscript. GLS was responsible for acquisition of the clinical information and reviewing the manuscript. All authors read and approved the final manuscript.

\section{Ethics approval and consent to participate}

Written informed consent was obtained from the patient's parents for the participation. A copy of the written consent is available for review by the Editor of this journal.

\section{Consent for publication}

The parent of the patient consented to the publication of the case and any accompanying images with written consent.

\section{Competing interests}

The authors declare that they have no competing interests.

\section{Publisher's Note}

Springer Nature remains neutral with regard to jurisdictional claims in published maps and institutional affiliations.

Received: 20 June 2017 Accepted: 3 October 2017

Published online: 06 October 2017

\section{Rerefences}

1. Kirstein L, Silfverskiold BP. A family with emotionally precipitated drop seizures. Acta Psychiatr Neurol Scand. 1958;33:471-6.

2. Shiang R, Ryan SG, Zhu YZ, Hahn AF, O'Connell P, Wasmuth JJ. Mutations in the alpha 1 subunit of the inhibitory glycine receptor cause the dominant neurologic disorder, hyperekplexia. Nat Genet. 1993:5:351-8.

3. Al-Owain M, Colak D, Al-Bakheet A, Al-Hashmi N, Shuaib T, Al-Hemidan A, et al. Novel mutation in GLRB in a large family with hereditary hyperekplexia. Clin Genet. 2012;81:479-4.

4. Chung SK, Bode A, Cushion TD, Thomas RH, Hunt C, Wood SE, et al. GLRB is the third major gene of effect in hyperekplexia. Hum Mol Genet. 2013;22: 927-40.

5. James VM, Bode A, Chung SK, Gill JL, Nielsen M, Cowan FM, et al. Novel missense mutations in the glycine receptor beta subunit gene (GLRB) in startle disease. Neurobiol Dis. 2013:52:137-49.

6. Lee CG, Kwon MJ, Yu HJ, Nam SH, Lee J, Ki CS, et al. Clinical features and genetic analysis of children with hyperekplexia in Korea. J Child Neurol. 2013;28:90-4.

7. Mine J, Taketani T, Otsubo S, Kishi K, Yamaguchi S. A 14-year-old girl with hyperekplexia having GLRB mutations. Brain and Development. 2013;35: 660-3.

8. Rees Ml, Lewis TM, Kwok JB, Mortier GR, Govaert P, Snell RG, et al. Hyperekplexia associated with compound heterozygote mutations in the beta-subunit of the human inhibitory glycine receptor (GLRB). Hum Mol Genet. 2002;11:853-60.

9. Rees Ml, Harvey K, Ward H, White JH, Evans L, Duguid IC, et al. Isoform heterogeneity of the human gephyrin gene (GPHN), binding domains to the glycine receptor, and mutation analysis in hyperekplexia. J Biol Chem. 2003;278:24688-96.

10. Harvey K, Duguid IC, Alldred MJ, Beatty SE, Ward H, Keep NH, et al. The GDP-GTP exchange factor collybistin: an essential determinant of neuronal gephyrin clustering. J Neurosci. 2004;24:5816-26.

11. Carta E, Chung SK, James VM, Robinson A, Gill JL, Remy N, et al. Mutations in the GlyT2 gene (SLC6A5) are a second major cause of startle disease. J Biol Chem. 2012:287:28975-85.

12. Eulenburg V, Becker K, Gomeza J, Schmitt B, Becker CM, Betz H. Mutations within the human GLYT2 (SLC6A5) gene associated with hyperekplexia. Biochem Biophys Res Commun. 2006;348:400-5.

13. Gimenez C, Perez-Siles G, Martinez-Villarreal J, Arribas-Gonzalez E, Jimenez E,

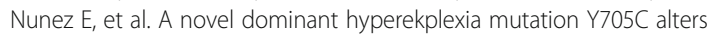

trafficking and biochemical properties of the presynaptic glycine transporter GlyT2. J Biol Chem. 2012;287:28986-9002.

14. Rees MI, Harvey K, Pearce BR, Chung SK, Duguid IC, Thomas P, et al. Mutations in the gene encoding GlyT2 (SLC6A5) define a presynaptic component of human startle disease. Nat Genet. 2006;38:801-6.

15. Winczewska-Wiktor A, Badura-Stronka M, Monies-Nowicka A, Nowicki MM, Steinborn B, Latos-Bieleńska $A$, et al. A de novo CTNNB1 nonsense mutation associated with syndromic atypical hyperekplexia, microcephaly and intellectual disability: a case report. BMC Neurol. 2016;16:35. doi:10.1186/s12883-016-0554-y.

16. Lee $Y$, Kim NY, Hong S, Chung SJ, Jeong SH, Lee PH, et al. Familiar Hyperekplexia, a potential cause of cautious gait: a new Korean case and a systematic review of phenotypes. J Mov Disord. 2017;10:53-8.

17. Bode A, Lynch JW. The impact of human hyperekplexia mutations on glycine receptor structure and function. Mol Brain. 2014;7:2. doi:10. 1186/1756-6606-7-2.

18. Richards CS, Bale S, Bellissimo DB, Das S, Grody WW, Hegde MR, et al. ACMG recommendations for standards for interpretation and reporting of sequence variations: revisions. Genet Med. 2007;10:294-300.

19. Zhou L, Chillag KL, Nigro MA. Hyperekplexia: a treatable neurogenetic disease. Brain and Development. 2002;24:669-74.

20. Thomas RH, Chung SK, Wood SE, Cushion TD, Drew CJ, Hammond CL, et al. Genotype-phenotype correlations in hyperekplexia: apnoeas, learning difficulties and speech delay. Brain. 2013:136:3085-95.

21. Villmann C, Oertel J, Melzer N, Becker CM. Recessive hyperekplexia mutations of the glycine receptor alpha1 subunit affect cell surface integration and stability. J Neurochem. 2009;111:837-47.

22. McKeon A, Martinez-Hernandez E, Lancaster E, Matsumoto JY, Harvey RJ, McEvoy KM, et al. Glycine receptor autoimmune spectrum with stiff-man syndrome phenotype. JAMA Neurol. 2013;70:44-50.

23. Hutchinson M, Waters P, McHugh J, Gorman G, O'Riordan S, Connolly S, et al. Progressive encephalomyelitis, rigidity, and myoclonus: a novel glycine receptor antibody. Neurology. 2008;71:1291-2.

\section{Submit your next manuscript to BioMed Central and we will help you at every step:}

- We accept pre-submission inquiries

- Our selector tool helps you to find the most relevant journal

- We provide round the clock customer support

- Convenient online submission

- Thorough peer review

- Inclusion in PubMed and all major indexing services

- Maximum visibility for your research

Submit your manuscript at www.biomedcentral.com/submit
Biomed Central 